\title{
Highs and Lows in English Attachment
}

\author{
Nino Grillo ${ }^{\mathrm{a}, *}$, João Costa $^{\mathrm{b}}$, Bruno Fernandes $^{\mathrm{b}}$, Andrea Santi $^{\mathrm{c}}$ \\ ${ }^{a}$ Institut für Linguistik: Anglistik, Universität Stuttgart \\ ${ }^{b}$ Centro de Linguística da Universidade Nova de Lisboa \\ ${ }^{c}$ Department of Linguistics, University College London
}

\section{Abstract}

Grillo \& Costa (2014) claim that Relative-Clause attachment ambiguity resolution is largely dependent on whether or not a Pseudo-Relative interpretation is available. Data from Italian, and other languages allowing Pseudo-Relatives, support this hypothesis. Pseudo-Relative availability, however, covaries with the semantics of the main predicate (e.g., perceptual vs. stative). Experiment 1 assesses whether this predicate distinction alone can account for prior attachment results by testing it with a language that disallows Pseudo-Relatives (i.e. English). Low Attachment was found independent of Predicate-Type. Predicate-Type did however have a minor modulatory role. Experiment 2 shows that English, traditionally classified as a Low Attachment language, can demonstrate High Attachment with sentences globally ambiguous between a Small-Clause and a reduced Relative-Clause interpretation. These results support a grammatical account of previous effects and provide novel evidence for the parser's preference of a SmallClause over a Restrictive interpretation, crosslinguistically.

Keywords: Sentence Processing, Parsing Universals, Attachment Preferences, Relative Clauses, Pseudo Relative Small Clauses.

\footnotetext{
${ }^{*}$ Corresponding author. Institut für Linguistik: Anglistik, Universität Stuttgart, Azenbergstr. 12, 70174, Stuttgart, Germany. Tel.: +49-(0)711-685-83120, Fax: +49-(0)711-685-83122.

Email addresses: nino@ifla.uni-stuttgart.de (Nino Grillo), jcosta@fcsh.unl.pt (João Costa), Preprint submitted to Cognition 


\section{Introduction}

2

3 ing in which crosslinguistic variation is grounded in language specific grammatical properties.

4 Crosslinguistic variation in parsing preferences that does not stem from a grammatical distinc-

5 tion poses challenges to theories of parsing (Fodor, 1998a,b). Indeed, the language dependent

6 preference for either high or low attachment of the Relative Clause (RC) in (1) (first observed by

7 Cuetos \& Mitchell 1988 and replicated by many others $)^{1}$ has generated extensive investigation,

8 given there was no known grammatical distinction until recent work by Grillo (2012) and Grillo

9 \& Costa (2014). Speakers of English show an overall Low Attachment (LA) preference (i.e., 10 attaching to the actress in (1)), while speakers of Spanish, i.a., demonstrate a High Attachment 11 (HA) preference (attaching to the maid in (1)).

(1) a. John saw $\left[D P 1\right.$ the $\left[{ }_{N P 1} \operatorname{maid}_{1}\right.$ of $\left[{ }_{D P 2}\right.$ the $\left[{ }_{N P 2} \underline{\operatorname{actress}_{2}}\left[{ }_{C P}\right.\right.$ that was 2 standing on the balcony $\left.\left.\left.]\right]\right]\right]$ b. Juan vio [DP1 la [NP1 criada $_{1}$ de [DP2 la [NP2 actriz $\left._{2}\right]$ [CP que estaba ${ }_{1}$ en el balcón $\left.\left.]\right]\right]$

Several earlier accounts for these results have captured some essential aspect of the phenomenon but not its entirety. Previous accounts include (i) assuming modification by RCs, and other non-primary relations, being parsed using a variety of non-structural principles (Gilboy et al., 1995); (ii) differences in frequency of exposure to HA vs. LA structures (Mitchell \& Cuetos, 1991); (iii) parametrization of parsing principles (Gibson et al., 1996), (iv) crosslinguistic differences in prosody (Fodor, 2002); and (v) crosslinguistic differences in the relativizing element (Hemforth et al., 1998). In more recent work, Hemforth et al. (2015) argues that crosslinguistic differences are more limited in scope than initially presumed and are largely based on

bruno86fernandes@gmail.com (Bruno Fernandes), a.santi@ucl.ac.uk (Andrea Santi) ${ }^{1}$ See Fernández (2003) for discussion of this vast literature. 
independent grammatical properties of the languages under scrutiny. Similarly, Grillo (2012) and Grillo \& Costa (2014) discuss a particular crosslinguistic grammatical variable that could explain the remaining variability: Pseudo-Relative (PR) availability.

Grillo (2012) and Grillo \& Costa (2014) identified a grammatical confound in the RC attachment literature: an asymmetric availability of Pseudo-Relatives (PR) across languages and structures. The PR is string identical to an RC, but the two differ from each other structurally and interpretively. RCs (1) modify Noun-Phrases (NPs) and denote properties of entities, while PRs (2-a) are either complements or adjuncts of Verb-Phrases (VPs) and denote events, much like the English eventive Small-Clause (SC) in (2-b), which is the closest English translation of (2-a) and should not be confused with the string-identical (reduced-)RC interpretation.

(2) a. Juan vio $\left[P R[D P\right.$ la criada 1 de la actriz 2$]\left[{ }_{C P}\right.$ que $_{1 / * 2}$ estaba $_{1 / * 2}$ en el balcón $\left.]\right]$ b. John saw $\left[S C\left[D P\right.\right.$ the $\operatorname{maid}_{1}$ of the actress 2$]\left[V P\right.$ standing ${ }_{1 / * 2}$ on the balcony $\left.]\right]$.

The relevance of PRs for RC-attachment comes from the fact that the PR reading is incompatible with LA: the highest NP is the only accessible subject for the embedded verb in this structure. Grillo \& Costa observe a tight correspondence between PR-availability and attachment preference where HA is observed when PRs are available and LA when only RCs are available. To explain this pattern, they propose that, all else being equal, PRs are preferred by the parser over RCs for their simpler structure and interpretive properties. This proposal, dubbed the PR-first Hypothesis is supported by the reanalysis of previous findings and by novel results from languages that allow PRs (see below).

After providing a detailed overview of the PR-first Hypothesis and the data that support it we present two experiments testing a non PR-language, English, in order to: (1) determine whether 
44 these earlier results can alternatively be explained by the pragmatics of the predicates that al-

45 low PRs and, after failing to support such a pragmatic account, (2) test the generalizability and

46 crosslinguistic nature of the grammatical claims made by PR-first in turning English, typically a

47 LA language, into a HA language through SC-availability (grammatically similar to PRs).

48 1.1. PR-first Hypothesis

Grillo (2012) and Grillo \& Costa (2014) build on the observation that PRs are both struc-

50 turally and interpretively simpler than RCs. Structurally, PRs (being SCs) have an impoverished

51 internal structure when compared to RCs: e.g. Tense is anaphoric in PRs but deictic in RCs. In-

52 terpretively, PRs provide information relevant to the matrix event (i.e. what is perceived), and are

53 thus preferred following Relativized Relevance (Frazier, 1990; Traxler \& Frazier, 2008). More-

54 over, PRs carry fewer unsupported presuppositions than RCs, as they do not require a contrast

55 set (Crain \& Steedman, 1985; Altmann \& Steedman, 1988).

${ }_{56}$ On the basis of these observations, Grillo and Costa propose the PR-first Hypothesis, which

57 states that PRs should be preferred by the parser over RCs. Given that HA is obligatory with

58 PRs, we should expect to observe HA to be more frequent in languages and structures that allow

59 PRs and LA with unambiguous $\mathrm{RC}$ readings. ${ }^{2}$

Support for these predictions comes from both reanalysis of previous results from the lit-

61 erature, which shows an almost perfect correspondence between PR-availability and attachment

62 preferences, and novel experimental results which directly manipulated PR-availability in a num-

63 ber of PR-languages including Italian (Grillo \& Costa, 2014), French (Grillo et al., 2015), Greek

64 (Grillo \& Spathas, 2014) and European Portuguese (Fernandes, 2012; Grillo et al., 2012, 2013;

65 Tomaz et al., 2014). PR-availability depends on a number of factors, but only a well-known

${ }^{2}$ Provided that other factors (e.g. prosody, referentiality) are controlled for. 
restriction on the properties of the matrix verb is relevant to this paper (for full discussion see

67 Cinque 1992).

68

Like eventive SCs in English, PRs denote events and need licensing via predicates that can take events as their complements, e.g. (semi)perceptual predicates, both verbal (see, hear) and nominal: (picture of). Stative/relational predicates (work for), and entity-denoting nominals

71 (house of), can only select for entities/NPs, and thus do not license PRs or eventive SCs, but are 72 perfectly acceptable with RC-modified NPs.

In an offline questionnaire in Italian, Grillo and Costa compared attachment preferences in minimal pairs of sentences. The sentences contained either a PR-compatible verb, being ambiguous between a PR/RC interpretation (3-a), or a stative verb and only permitting an RC interpretation (3-b).

77

Contrary to (3-b), (3-a) is ambiguous between a PR-reading, in which the whole clause denotes the direct perception of an event (the grandma screaming) and the RC reading, in which the matrix clause denotes the perception of an individual (the grandma) and the embedded clause denotes a modifier of either of the two NPs (the unique grandma/girl that screamed).

\section{Example stimuli from Experiment II (Grillo \& Costa, 2014)}

a. $\quad \mathrm{PR} / \mathrm{RC}$ CONDITION

Maria ha sentito la nonna della ragazza che gridava.

M. heard the grandma of the girl that was screaming.

b. RC-ONLY CONDITION

Maria lavora con la nonna della ragazza che gridava.

M. works with the grandma of the girl that was screaming. 
In line with the predictions of $P R$-first, the results show a strong preference for HA in the ambiguous $\mathrm{PR} / \mathrm{RC}$ condition (78.6\% HA) and a strong LA preference with unambiguous RCs $90 \quad(24.2 \% \mathrm{HA})$.

${ }_{91}$ In this paper, we explore an alternative explanation for this result. The effects described above could equally be due to the predicate semantics, which covaries with PR-availability. Event-

9з taking "PR-predicates" may simply favour HA for reasons other than PR-availability, namely 94 plausibility. Consider the extreme case of the PR-predicate 'interrupt' in "John interrupted the maid of the actress that was talking". This sentence has a clear HA bias as the person interrupted 96 (NP1) was reasonably also the person who was talking. A similar account could in principle explain the reported HA-bias with other PR-predicates like perceptual verbs. Modulation of RCattachment through pragmatics was demonstrated by Gilboy et al. (1995). More specific effects

of matrix verb type in RC-attachment have recently been observed by Rohde et al. (2011), who showed that implicit causality verbs strongly influence RC-attachment: higher proportions of HA were observed with 'detest' as a matrix verb in the following contrast: John detests/babysits the children of the musician who ... when the RC provided an explanation for the state of affair described in the matrix clause. Taken together, these results justify testing an alternative, pragmatic account.

Importantly, a semantic/pragmatic account of the effect of Verb-Type observed by Grillo and Costa would predict the manipulation of perceptual vs. stative verbs to produce the same attachment distinction in English as has been observed in PR-languages. This was tested in Experiment 1. 


\section{Experiment 1: Verb-type effects}

30 monolingual British English speakers participated in a timed questionnaire after giving their informed consent.

Materials and Design 24 sets of target sentences (4) were constructed, in a 2(PredicateType: SC-compatible vs. RC-only)*2(Environment: Verbal vs. Nominal) Latin-square design with 70 unrelated fillers. The complex NP+RC was kept identical across conditions. Sentences in the verbal condition are translated from Grillo \& Costa (2014). Verbs in the SC-compatible condition were cognates of those used in the original Italian experiment. A few adaptations were necessary in the RC-only condition when cognates were not available or would have generated non-natural sentences. Whenever a change of verb was necessary, we closely matched its semantic and syntactic properties to those of the original verb. ${ }^{3}$ Contrasting Verbal and Nominal predicates, replicates design features of similar previous studies by Fernandes (2012) and Grillo et al. (2012), and allows for a better evaluation of the effects of SC-availability across syntactic positions.

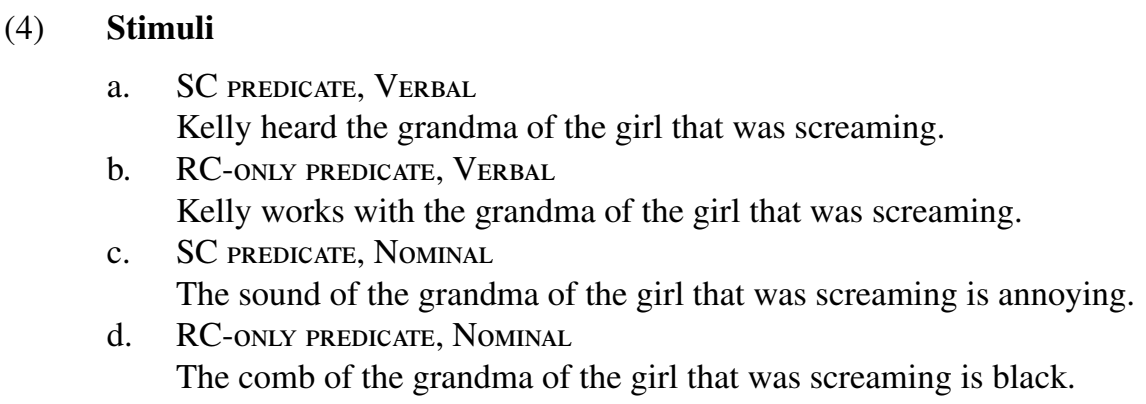

\footnotetext{
${ }^{3}$ The original study also included two psych-verbs (hate/love) in the RC-only condition. These verbs can in fact also introduce PR/SCs, albeit more marginally, and were thus avoided in the present study.
} 
${ }_{132}$ Stimuli were presented using Linger (http://tedlab.mit.edu/ dr/Linger/) in soundproof booths

${ }_{133}$ in UCL. After each sentence, participants were prompted to select one of two alternative sen-

${ }_{134}$ tences (e.g. the grandma screamed/the girl screamed). Sentences were pseudo-randomized and

135 the order of presentation of High/Low attachment choices was counterbalanced. See Appendix

${ }_{136}$ for full list of stimuli.

137 2.0.1. Analysis

${ }_{138}$ A mixed effects model computed on the attachment preferences, with Predicate-Type and

139 Environment as fixed factors and Subject and Item as random factors, and random slopes for fixed

140 effects and their interactions, showed a highly significant effect of Predicate-Type $(p<.001)$, with

141 more HA preferences for event-taking than entity-taking predicates; no effect of Environment

$142(p=.6)$ and no interaction $(p=.2)$.

143

\begin{tabular}{cccl}
\hline NP-RC & NP-SC & VP-RC & VP-SC \\
\hline 28.4 & 32.5 & 19.5 & 37.2
\end{tabular}




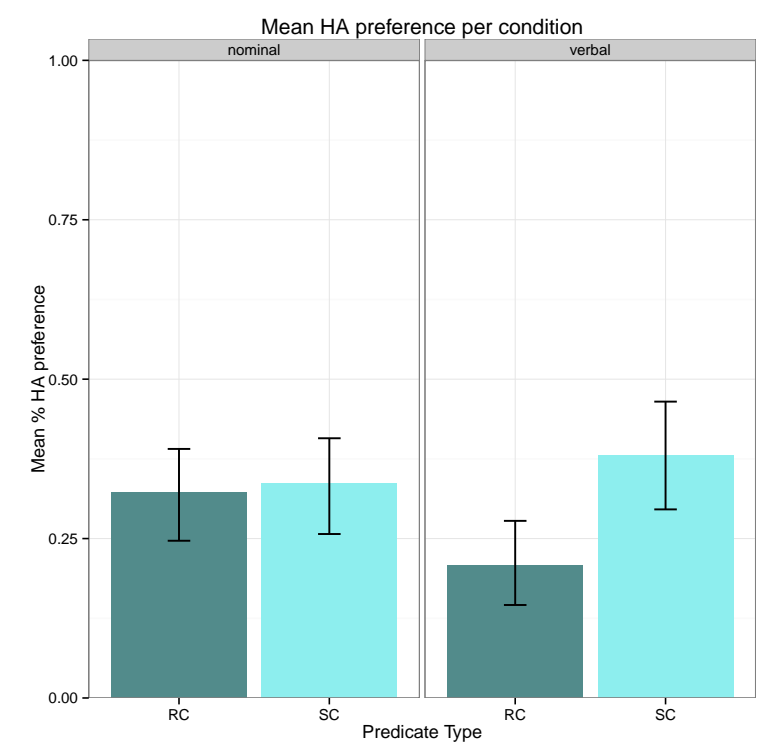

Figure 1: Mean HA preferences Experiment 1 (cf. \% of HA in original Italian experiment: RC 24.2\% /PR: 78.6\%)

\begin{tabular}{lcccc}
\hline contrast & coefficient & SE & z-value & $\mathrm{p}$ \\
\hline Predicate-Type & 0.7387 & 0.2183 & 3.384 & $0.000714^{* * *}$
\end{tabular}
observed, as predicted by $P R$-first and in contrast to what was observed in Italian and other PR-languages. The strong LA preference with the entity-taking predicates, on the other hand, matches results in Italian with the same predicates. This is supported by the results of a mixed effect model with Language as fixed factor, which revealed a significant interaction between Verb-Type and Language $(\mathrm{p}<.001)$. The Language effect is limited to perceptual verbs $(\mathrm{p}<.001)$, and completely absent in the RC-only condition ( $\mathrm{p}=.995)$. The effect of Predicate-Type in English, which might be attributed to an increased plausibility of a HA continuation with perceptual verbs, is of a significantly smaller magnitude than what was observed in Italian. The results are

154 incompatible with a semantic/pragmatic account of previous findings in PR-languages. 
Having established that plausibility alone cannot account for the results from Italian (and other PR-languages), in Experiment 2 we test whether the PR-first Hypothesis can be generalized to make predictions about non-PR languages by using reduced relative clauses in English, which provide an ambiguity between RC and SC interpretations, comparable to the PR/RC ambiguity.

The embedded gerund (5-a) (screaming) is ambiguous between a reduced-RC-reading and an eventive SC-reading. This ambiguity disappears in (5-b), where the embedded predicate can only be interpreted as a reduced-RC.

(5) a. Kelly heard the grandma of the girl screaming.

b. Kelly works with the grandma of the girl screaming.

Following the rationale of $P R$-first, we would expect a clear preference for HA with (5-a), while an overall LA preference should still be observed with (5-b). To test this, Experiment 2 modified the stimuli from Experiment 1 to generate reduced RCs.

\subsection{Experiment 2: Testing SC-availability in English}

This experiment generalizes the predictions of $P R$-first to English, by comparing attachment in ambiguous SC/Reduced-RC sentences (i.e., event-taking predicates) with unambiguous Reduced-RCs-only sentences (entity-taking predicates).

Materials and Design 30 native British-English speakers were tested with stimuli from Experiment 1 with the following change: we removed the complementizer and auxiliary of the relative clause to generate Reduced-RCs or SCs (depending on the predicate present). With event-taking predicates the relevant string (the $x$ singing) was globally ambiguous between an SC and a Reduced-RC-parse but only allowed a Reduced-RC reading under entity-taking predicates. 


\begin{tabular}{cccc}
\hline NP RC & NP SC & VP RC & VP SC \\
\hline 13.8 & 56.1 & 21.1 & 55.5
\end{tabular}

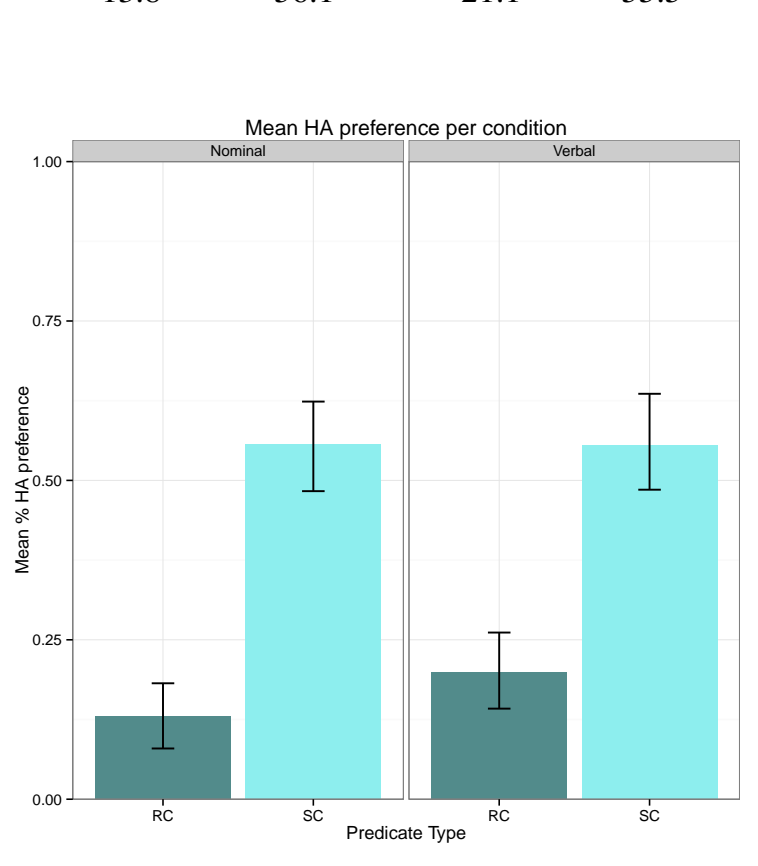

Figure 2: Mean HA preferences Experiment 2 with event-taking predicates, but LA with entity-taking predicates.

\subsection{Results} in SC-compatible contexts (i.e. above 50\%), and a strong LA preference in RC-only contexts.

We acknowledge that Reduced-RCs might not be the easiest structure to parse, but this cost should be independent of attachment. Contrary to Experiment 1, PR-first predicts overall HA

The same analysis as in Experiment 1 was used. As predicted, we observed a HA preference

A strongly significant effect of predicate-type $(p<.0001)$ was observed, with greater HA preference for event-taking than entity-taking predicates. There was no significant effect of environment $(p=.5)$ and no interaction between the two factors $(p=.3)$. 


\begin{tabular}{lcccc}
\hline contrast & coefficient & SE & z-value & $\operatorname{Pr}(>|\mathrm{z}|)$ \\
\hline Predicate-Type & 2.3894 & 0.3238 & 7.380 & $1.58 \mathrm{e}-13 * * *$
\end{tabular}

In order to provide a statistical test of the greater effect of grammar over predicate semantics, we ran a mixed model logistic regression adding Experiment[1 vs. 2] to Predicate-type and Environment as fixed factors, with random slopes and intercepts fit for the fixed effects.

Besides a main effect of Predicate-Type $(p<.001)$, the analysis indicated both a significant 2way Predicate-Type*Experiment interaction $(p<.001)$ and a 3 way Predicate-type*Environment*Experiment interaction $(p<.05)$. The 2 way interaction is due to a higher proportion of HA in the PRcompatible condition in Experiment 2 than 1. The 3 way interaction is due to a significantly higher proportion of HA in the nominal environment in Experiment 1 than 2 in the RC-only condition. This effect might be attributed to the relative length of the RC in the two experiments (longer RCs in Experiment 1 than 2), i.e. as an effect of implicit prosody. It has been demonstrated that longer RCs display a stronger tendency for HA than shorter RCs (Fodor, 2002; Hemforth et al., 2015, among others). Notice, however, that the effect goes in the opposite direction with SC-compatible, event-taking predicates. We take this as evidence that both Prosody and $\mathrm{PR} / \mathrm{SC}$-availability are grammatical factors involved in the resolution of attachment ambiguities.

\begin{tabular}{lcccc}
\hline contrast & coefficient & SE & z-value & $\operatorname{Pr}(>|\mathrm{z}|)$ \\
\hline Predicate-Type & 1.50065 & 0.18544 & 8.092 & $5.85 \mathrm{e}-16 * * *$ \\
Predicate-Type*Experiment & -1.55802 & 0.36040 & -4.323 & $1.54 \mathrm{e}-05 * * *$ \\
Predicate-Type*Environment*Experiment & 1.25753 & 0.56927 & 2.209 & $0.0272 *$
\end{tabular}

\section{General Discussion}

The best evidence to date in favour of $P R$-first comes from data contrasting RC-attachment preferences under event-taking predicates (PR-compatible), which drive strong HA preference in PR-languages, and entity-taking (RC-only) predicates, which, on the contrary, drive a strong LA preference in the same languages (Grillo \& Costa, 2014, and work cited above). We pointed 
out that an exclusively pragmatic account of the attachment preferences is viable in principle, as PR-availability covaries with the semantic properties of the matrix verb. Rohde et al. (2011) have already shown that properties of the matrix verb can play an important role in shaping attachment preferences. Implicit causality verbs trigger HA preference in languages in which a strong LA preference is generally observed (Rohde et al., 2011).

Experiment 1 was designed to test this alternative account in a non-PR language, English, so as to avoid conflating the effect of the two factors. The experiment is a close replication of an Italian experiment (Experiment 2 in Grillo \& Costa 2014), which showed a strong attachment asymmetry between sentences containing event-taking (78.6\% HA) vs. entity-taking predicates (24.2\% HA). An additional manipulation was tested in English, nominal vs. verbal domain. Experiment 1, however, did not replicate the findings from Italian: while predicate semantics appeared to play a minor modulatory role in attachment, a generalized LA preference was found across both conditions (nominal and verbal). This argues against the alternative pragmatic account.

Experiment 2 replicated Experiment 1, but used reduced RCs instead of full RCs. Reduced RCs under event-taking predicates demonstrate a similar type of grammatical ambiguity (SC vs. $\mathrm{RC}$ ) as found in PR-languages (PR vs. RC). SC-availability leads to a change from LA to HA in English, mirroring the Italian results under PR-availability. SC-availability resulted in the same attachment effects across both nominal and verbal environments, which further shows the primacy of this factor in determining attachment preferences. A strong LA preference, which corresponds to the Italian results in the same environment, emerges in unambiguous RC-only contexts in both Experiment 1 and 2. These results further illustrate the strength of locality principles in attachment when SC-availability is controlled for.

Collectively, these and previous results from similar experiments on PR-languages, show that 
attachment preferences are not language dependent, but rely heavily on (universal) grammatical factors, such as the availability of an eventive SC interpretation (among other universal factors such as prosody and referentiality). The results advocate for a crucial role for syntactic structure above and beyond the semantic plausibility of HA with SC-predicates.

Finally, the results from Experiment 2 require that PR-first be framed in more general terms of eventive clauses rather than specific constructions (i.e. PRs). Likewise, there are no longer HA and LA languages, but grammatical environments that favour HA or LA. Crucially, parsing preferences are equivalent across languages.

Acknowledgements This research is part of the project Syntactic and lexical factors in processing complexity funded by the Fundação para a Ciência e a Tecnologia with the research grant PTDC/CLE-LIN/114212/2009 awarded to Nino Grillo. We gratefully acknowledge the FCT contribution. Experiment 1 and 2 were originally presented at the 26th CUNY Conference on Human Sentence Processing in Columbia (SC) and the 25th AMLaP (Architectures and Mechanisms on Language Processing) in Edinburgh (UK). We thank the organizers, reviewers and participants for useful comments and suggestions. Thanks to Masaya Yoshida, Jennifer Arnold and two anonymous Cognition reviewers for insightful comments on an earlier version of this manuscript. 
Altmann, G. T. M., \& Steedman, M. (1988). Interaction with context during human sentence processing. Cognition, 30, $191-281$.

Cinque, G. (1992). The Pseudo-Relative and Acc-ing constructions after verbs of perception. In University of Venice Working Papers in Linguistics. Università di Venezia.

Crain, S., \& Steedman, M. (1985). On not being led up to garden path: The use of context by the psychological parser. In D. Dowty, L. Karttunen, \& A. Zwicky (Eds.), Natural language processing: Psychological, computational, and theoretical perspectives. Cambridge University Press.

Cuetos, F., \& Mitchell, D. C. (1988). Cross-linguistic differences in parsing. Cognition, 30, 73-105.

Fernandes, B. (2012). Attachment Preferences in Prepositional Infinitive Constructions. Master's thesis Centro de Linguística da Universidade Nova de Lisboa.

Fernández, E. (2003). Bilingual sentence processing: Relative clause attachment in English and Spanish. Amsterdam: John Benjamins.

Fodor, J. D. (1998a). Learning to Parse? Journal of Psycholinguistic Research, 27, 285-319.

Fodor, J. D. (1998b). Parsing to Learn? Journal of Psycholinguistic Research, 27, 339-374.

Fodor, J. D. (2002). Prosodic disambiguation in silent reading. In M. Hirotani (Ed.), Proceedings of the North East Linguistic Society. GSLA, University of Massachusetts, Amherst.

Frazier, L. (1990). Parsing modifiers. Special purpose routines in the human sentence processing mechanism? In D. Balota, \& G. F. d'Arcais (Eds.), Comprehension Processes in Reading (pp. 303-330). Hillsdale, N.J.: Lawrence Erlbaum.

Gibson, E., Pearlmutter, N., Canseco-Gonzalez, E., \& Hickok, G. (1996). Recency preference in the human sentence processing mechanism. Cognition, 59, 23-59.

Gilboy, E., Sopena, J., Clifton, C., \& Frazier, L. (1995). Argument structure and association preferences in Spanish and English complex NPs. Cognition, 54, 131-167.

Grillo, N. (2012). Local and universal. In V. Bianchi, \& C. Chesi (Eds.), Enjoy Linguistics! Papers offered to Luigi Rizzi on the occasion of his 60th birthday (pp. 234-245). Siena, Italy: CISCL Press.

Grillo, N., \& Costa, J. (2014). A novel argument for the universality of parsing principles. Cognition, 133, $156-187$.

Grillo, N., Fernandes, B., \& Costa, J. (2012). Attachment preferences in Prepositional Infinitive Constructions in European Portuguese. In AMLaP (Architectures and Mechanisms for Language Processing). Riva del Garda, Italy.

Grillo, N., Hemforth, B., Pozniak, C., \& Santi, A. (2015). Pseudo Relatives are easier than Relative Clauses: Evidence from Tense. In E. Kaiser, T. Mintz, R. Pancheva, \& J. Zevin (Eds.), 28th CUNY Conference on Human Sentence 
Grillo, N., \& Spathas, G. (2014). Tense and Aspect modulate RC attachment: Testing the PR hypothesis in Greek. In Experimental and theoretical approaches to relative clauses reconciled. Marburg, Germany: 36 DGfS (Jahrestagung der Deutschen Gesellschaft für Sprachwissenschaft).

Grillo, N., Tomaz, M., Lourenço Gomes, M., \& Santi, A. (2013). Pseudo relatives vs. Relative clauses: Greater preference, Lower costs. In AMLaP (Architectures and Mechanisms for Language Processing). Marseille, France.

Hemforth, B., Fernández, S., Clifton, C., Frazier, L., Konieczny, L., \& Walter, M. (2015). Relative clause attachment in German, English, Spanish and French: Effects of position and length. To appear in [Lingua].

Hemforth, B., Konieczny, L., Scheepers, C., \& Strube, G. (1998). Syntactic ambiguity resolution in German. In D. Hillert (Ed.), Syntax and Semantics: A cross-linguistic perspective (pp. 293-312). San Diego: Academic Press.

Mitchell, D. C., \& Cuetos, F. (1991). The origin of parsing strategies. In C. Smith (Ed.), Current issues in Natural Language Processing (pp. 1-12). Center for Cognitive Science, U. of Austin, TX.

Rohde, H., Levy, R., \& Kehler, A. (2011). Anticipating explanations in relative clause processing. Cognition, 118, 339-358.

Tomaz, M., Lourenço Gomes, M., Santi, A., \& Grillo, N. (2014). A concordância de número em construções relativas e pseudorelativas em português europeu. In Textos Selecionados do XXIX Encontro Nacional da Associação Portuguesa de Linguística.

Traxler, M. J., \& Frazier, L. (2008). The role of pragmatic principles in resolving attachment ambiguities: Evidence from eye movements. Memory and Cognition, 36, 314-328.

\section{Appendix A. Experimental Items}

List of stimuli for Experiment 1 and 2, material in parentheses (that was) was omitted in

Experiment II. Mean \% of HA is indicated for each item.

\section{Sentences}

Experiment 1/2

1. a. Jim saw the son of the doctor (that was) having dinner.

b. The picture of the son of the doctor (that was) having dinner is old. 
c. Jim shares the house with the son of the doctor (that was) having dinner.

3. a. John heard the teacher of the boy (that was) singing.

b. The film of the teacher of the boy (that was) singing is of low quality.

4. a. The writer watched the aunt of the girl (that was) jumping.

b. The drawing of the aunt of the girl (that was) jumping is pretty.

b. The scene of the friend of the politician (that was) cooking is long. 
7. a. Jane caught the maid of the actress (that was) stealing.

b. The sight of the maid of the actress (that was) stealing is horrible.

c. Jane trains with the maid of the actress (that was) stealing.

d. The scarf of the maid of the actress (that was) stealing is horrible.

$33.3 / 14.2$

8. a. The lawyer caught the chauffeur of the neighbour (that was) swimming.

$16.6 / 71.4$

b. The video of the chauffeur of the neighbour (that was) swimming is boring.

$0 / 62.5$

c. The lawyer exercises with the chauffeur of neighbour (that was) swimming.

$14.2 / 28.5$

d. The dog of the chauffeur of the neighbour (that was) swimming is smelly.

$66.6 / 12.5$

9. a. David observed the son of the maid (that was) exercising.

$44.4 / 62.5$

b. The footage of the son of the maid (that was) exercising is missing.

c. Mary is divorced from the son of the maid (that was) exercising.

d. The wallet of the son of the maid (that was) exercising is missing.

10. a. Alan observed the nephew of the nurse (that was) eating.

b. The image of the nephew of the nurse that eating is amusing.

c. Alan relates to the nephew of the nurse that eating.

d. The life of the nephew of the nurse (that was) eating is amusing.

11. a. Peter photographed the co-worker of the butcher (that was) running.

b. The sound of the co-worker of the butcher (that was) running is disturbing. $\quad 57.1 / 85.7$

c. Peter trains with the co-worker of the butcher (that was) running.

$44.4 / 0$

d. The moustache of the co-worker of the butcher (that was) running is disturbing. 50/14.2

12. a. Kate looked at the friend of the judge (that was) driving.

$50 / 57.1$

b. The recollection of the friend of the judge (that was) driving is fuzzy. 
c. Kate is engaged to the friend of the judge (that was) driving.

b. The noise of the friend of the flower girl (that was) working is unbearable.

$16.6 / 71.4$

c. Lily parties with the friend of the flower girl (that was) working.

$0 / 12.5$

d. The toothache of the friend of the flower girl (that was) working is unbearable. 14.2/28.5

14. a. Rachel dreamt of the friend of the brother (that was) drinking.

b. The scene of the friend of the brother (that was) drinking is sad.

c. Rachel is married to the friend of the brother (that was) drinking.

$16.6 / 14.2$

d. The office of the friend of the brother (that was) drinking messy.

$16.6 / 12.5$

15. a. David drew the grandson of the woman (that was) smoking.

b. The depiction of the grandson of the woman (that was) smoking is ugly.

c. David is employed by the grandson of woman (that was) smoking.

$11.1 / 0$

d. The watch of the grandson of the woman (that was) smoking is ugly.

$16.6 / 0$

16. a. Phillip filmed the agent of the player (that was) snoring.

$66.6 / 71.4$

b. The sound of the agent of the player (that was) snoring is terrible.

$66.6 / 50$

c. Phillip hangs out with the agent of the player (that was) snoring.

d. The t-shirt of the agent of the player (that was) snoring is terrible.

17. a. The fireman recorded the cousin of the lawyer (that was) whistling.

b. The portrayal of the cousin of the lawyer (that was) whistling is lovely.

c. The fireman is employed by the cousin of the lawyer (that was) whistling. 
18. a. Bob glanced at the friend of the shoemaker (that was) dancing.

b. The energy of the friend of the shoemaker (that was) dancing is amazing.

c. Bob is engaged to the friend of the shoemaker (that was) dancing.

$50 / 14.2$

d. The pool of the friend of the shoemaker (that was) dancing is amazing.

$0 / 12.5$

19. a. Sally photographed the stepson of the nurse (that was) studying.

b. The idea of the stepson of the nurse (that was) studying is surprising.

c. Sally collaborates with the stepson of the nurse (that was) studying.

$55.5 / 12.5$

d. The advice of the stepson of the nurse (that was) studying is surprising.

$50 / 14.2$

20. a. The singer watched the brother of the CEO (that was) bleeding.

$50 / 85.7$

b. The memory of the brother of the CEO (that was) bleeding is uncomfortable. 16.6/37.5

c. The singer studies with the brother of the CEO (that was) bleeding.

$42.8 / 14.2$

d. The couch of the brother of the CEO (that was) bleeding is uncomfortable.

$44.4 / 25$

21. a. The policeman filmed the friend of the sister (that was) sewing.

b. The vision of the friend of the sister (that was) sewing is boring.

c. The policeman is married to the friend of the sister (that was) sewing.

d. The work of the friend of the sister (that was) sewing is boring.

22. a. The architect imagined the sister of the colleague (that was) dancing.

$28.5 / 42.8$

b. The sight of the sister of the colleague (that was) dancing is extraordinary.

$66.6 / 75$

c. The architect is divorced from the sister of the colleague (that was) dancing. 16.6/28.5

d. The mansion of the sister of the colleague (that was) dancing is extraordinary.

$0 / 25$

23. a. David saw the teacher of the friend (that was) driving.

b. The film of the teacher of the friend (that was) driving is disturbing. 
c. The neighbour attends university with the son of the doorman (that was) singing. $28.5 / 28.5$

d. The car of the son of the doorman (that was) singing is ugly. 